\title{
Todos pueden aprender
}

\section{Everyone can learn}

\author{
María Teresa Bañuelos Hernández ${ }^{1}$ \\ Andrea Granados Gil² \\ Ingrid Giselle Uribe Castañeda ${ }^{3}$
}

\begin{abstract}
Resumen
En este documento se encuentran los resultados de la aplicación de un proyecto socioeducativo por parte de las maestras en formación enfocado a la inclusión, el cual da a conocer la importancia de la sensibilización y conocimiento hacia las diferentes discapacidades. El proyecto se enfocó hacia los alumnos de un Jardín de Niños con edades de entre 3 a 6 años extendiéndose a la comunidad circundante al preescolar. Se aplicaron actividades donde los alumnos experimentaban situaciones que viven diariamente las personas con capacidades diferentes, luego por medio de profesionales en lenguaje signado, se les enseñó algunas palabras para poder comunicarse con personas sordas, por mencionar algunas de las actividades aplicadas. Los resultados dieron a conocer la importancia de tener una sociedad inclusiva, comenzado por los niños y creando generaciones con servicio, empatía y respeto hacia la diversidad.
\end{abstract}

\footnotetext{
${ }^{1}$ María Teresa Bañuelos Hernández. Institución Benemérita y Centenaria Escuela Normal del Estado de Chihuahua Prof. Luis Urías Belderráin, México. Correo electrónico: maribanuelos64@gmail.com ID: http://orcid.org/0000-0001-5300-971X
}

${ }^{2}$ Andrea Granados Gil. Institución Benemérita y Centenaria Escuela Normal del Estado de Chihuahua Prof. Luis Urías Belderráin, México. Correo electrónico: andreagranadosgil@gmail.com

ID: http://orcid.org/0000-0002-5304-2103

${ }^{3}$ Ingrid Giselle Uribe Castañeda. Institución Benemérita y Centenaria Escuela Normal del Estado de Chihuahua Prof. Luis Urías Belderráin, México. Correo electrónico: ingriduc97@gmail.com

ID: http://orcid.org/0000-0002-6024-6788 
RECIE. Revista Electrónica Científica de Investigación Educativa Vol. 4, núm. 2, enero-diciembre 2019, pp. 1029-1037.

\title{
Palabras clave
}

Inclusión, empatía, sensibilización, comunidad, discapacidad.

\begin{abstract}
In this document we find the results of the application of a socio-educational project focused on inclusion. Where it makes us aware of the importance of awareness and knowledge towards different disabilities. It is focused on kindergarten students aged 3 to 6 years. The project was extended to the community surrounding the preschool. The activities were applied where students experienced situations that people with different abilities lived daily, then by means of professionals in signed language, they were taught some words to be able to communicate with deaf people, to mention some of the activities applied. The results made known the importance of having an inclusive society, started by children and creating generations with service, empathy and respect for diversity.
\end{abstract}

\section{Keywords}

Inclusion, empathy, sensibilization, community, disability.

\section{Introducción}

En este documento se presentan los resultados del proyecto Socioeducativo Todos pueden aprender que se llevó a cabo en el preescolar durante la jornada de prácticas docentes que se imparte en la institución. Lo anterior enfocado a dar solución a una problemática en el Jardín de Niños: la falta de la cultura de inclusión en los alumnos. Durante el periodo de tiempo en el que se llevaron a cabo las prácticas, se percato que los alumnos tenían conductas agresivas entre ellos, además de apáticas.

Lo anterior desencadenó una serie de interrogantes: ¿de dónde viene esa cultura de violencia?, ¿por qué no hay preocupación entre los mismos niños? Y finalmente si llegaran niños con capacidades diferentes al salón, ¿sabrían cómo tratarlo?, ¿lo ayudarían?, ¿se podría lograr un ambiente inclusivo? Esto fue el desencadenante hacia una posible mejora enfocada a sensibilizar tanto a los alumnos como a la comunidad aledaña al Jardín de Niños. Por ende, se investigó su contexto y de esta forma comenzar la investigación.

\section{Contexto}

Las prácticas se realizaron en un Jardín de Niños ubicado al norte de la ciudad de Chihuahua. La comunidad cuenta con tiendas de abarrotes 
cercanas, una primaria aledaña al Jardín, iluminación peatonal, drenaje, calles pavimentadas, ferreterías, una iglesia, parques, por mencionar algunos servicios. Dicho plantel cuenta con servicios como: agua, luz, internet, drenaje, teléfono, entre otros. El nivel socioeconómico de la comunidad es medio-bajo en la mayoría de las familias, los padres son mayormente empleados de maquiladoras cercanas a la zona o amas de casa en el caso de las madres.

En esta institución se encuentran seis maestras Licenciadas en Educación Preescolar, USAER (Unidad de Servicios de Apoyo a la Educación Regular) con profesores de Comunicación/lenguaje, psicomotricidad, psicología, trabajo social y un director de estas áreas. También hay una maestra de educación física y un profesor de música, además de dos intendentes y la directora del Jardín. Está formado por doce espacios, incluidos dirección, sala de juntas, USAER, biblioteca, aula de música y educación física, baños, un salón de primer año, dos de segundo y tres de tercero (cada grupo se constituye de entre 20 a 25 alumnos). Se supuso que, al ser un contexto de nivel medio-bajo no había conocimientos acerca de la cultura de la inclusión, podría ser que la falta de cultura derivada del contexto fuera la causante del conflicto antes mencionado, por lo anterior la animación sociocultural fue el paradigma elegido por los integrantes del grupo, llegando a la conclusión de que este serviría para realizar un cambio en la cultura del preescolar. José Luis Muñoz en su artículo "La cultura en la sociedad actual" cita a Edward B. Taylor, el cual nos menciona que

La cultura es todo aquel complejo que incluye conocimientos, costumbres, capacidades y hábitos adquiridos y la animación sociocultural es un instrumento que utiliza la democracia cultural para fomentar acción y crear agentes de cambio que trabajen junto con los destinatarios (Taylor, 1871).

\section{Diagnóstico}

Para comprobar que esta problemática fuera realmente relevante se buscó elaborar un buen diagnóstico en el cual la observación dio pie para la reflexión relacionada con la problemática detectada, y de esta manera conocer el verdadero conflicto que se enfrenta y el conocimiento previo de la sociedad del preescolar, pues es información de primera mano. Ya que "A pesar del colectivo de personas con discapacidad, no ha sido objeto prioritario de estudio ni de desarrollos específicos de política social que den respuesta a sus necesidades". (Verdugo, 2017). 
De entre varios mecanismos de recolección de datos se utilizó la entrevista, con la finalidad de entender el mundo desde la perspectiva del entrevistado y corroborar lo observado durante las jornadas de prácticas. Se entrevistó a 15 alumnos de manera informal e individualmente, para así saber si conocen o han conocido personas con alguna deficiencia ya sea visual, auditiva, física o cognitiva, y, en caso de ser así, cómo trataban a estas personas.

Una vez realizadas, se interpretaron los resultados para poder llegar a una conclusión y tener un punto de partida para el proyecto. Esto fue de gran utilidad para recabar los datos necesarios en el problema propuesto, ayudó a que la información obtenida fuera más completa y conocer las respuestas de los alumnos Toda esta información sirvió para aclarar dudas sobre cómo ven los entrevistados la problemática, asegurando respuestas más completas.

Las respuestas se clasificaron por su similitud, comprobando que realmente son muy pocos los que conocen cómo convivir con personas discapacitadas. Por lo que se optó por llevar a cabo actividades de sensibilización a los alumnos, creando un ambiente inclusivo, empático y preparado para cualquier situación que pudiera presentarse en un futuro en su vida cotidiana. Gracias a esto se redactó lo siguiente:

\section{Problemática}

Gracias a este análisis, se determinaron las problemáticas más importantes que dieron la oportunidad de elaborar el proyecto para la mejora de la institución. Entre los resultados más significativos encontrados por este medio, estuvieron la falta de empatía en los alumnos y conocimiento hacia las personas con discapacidad.

\section{Objetivos}

Lograr la sensibilización de a la comunidad entera, en la cual se incluye a padres de familia, alumnos y personal del Jardín de Niños para que tuvieran un pensamiento inclusivo, utilizando instrumentos especializados, actividades y visitas en las que se trabajó junto a los participantes de este proyecto.

\section{Metodología}

Las actividades lúdicas según el Programa de Estudio 2011 Preescolar "son actividades basadas en el juego, que deben tener distintos niveles de complejidad, para conocer los logros de los niños, potencialidades y 
habilidades". Por lo tanto, el proyecto se desarrolló con el objetivo de atraer a los alumnos al tema, creando aprendizajes creativos y divertidos que llamen su atención.

De acuerdo con lo anterior, se realizaron durante cinco días una serie de actividades relacionadas a reforzar el término de inclusión para que así se tuviera más empatía y una mejor relación, tanto en el Jardín como fuera de este. Pretendiendo que al concluir se fomentara un ambiente de inclusión y respeto entre los integrantes del preescolar y por ende la comunidad en el que está inmerso.

Para ello se utilizó un análisis con la técnica de FODA, y de esta manera reconocer con qué recursos contábamos y cuidar los detalles de atención que podrían afectar al proyecto. A su vez, se aplicaron diversos cuestionarios con el propósito de saber si las actividades que se estuvieron aplicando a lo largo de la jornada, tuvieron una función positiva y fueron de impacto para los alumnos, planteándoles preguntas concretas que nos proporcionen la información viable para valorar los resultados, lo que sirvió para conocer los aprendizajes adquiridos y los diferentes puntos de vista de los alumnos, respecto a las actividades y el desarrollo de las situaciones que se presenten, y determinar si funcionaron o no las labores desarrolladas.

El primer día se realizó un "rally" en el cual se vio la discapacidad visual. Por ello el recorrido fue sencillo pasar por aros y en zigzag por un camino de conos, en parejas un niño se vendó los ojos y otro lo guio por la mitad del recorrido. En este punto la venda se cambió y para ser el otro niño el que guiaría.

Para continuar, se les habló del autismo poniéndoles un cuento relacionado a ello, "El pato azul" basado en videos del mismo nombre, después se les pidió que dibujaran el tema de la historia mientras se hizo ruido con maracas, música fuerte, entre otros. Lo anterior para imitar algunos de los síntomas del espectro autista.

Luego se abordaron las actividades normales para ellos sin que se levantaran de su silla y otras sin el uso de una mano. De esta forma se abordó la discapacidad física. La cuarta actividad planeada fue una plática hecha por el Instituto Chihuahuense de Lengua de Señas Mexicana, en ella se les habló a los niños de las discapacidades auditivas. De esta manera se les enseñó el abecedario y algunas frases simples en lenguaje signado.

A manera de conclusión se les llevó a una visita al Centro de Rehabilitación Infantil Teletón (CRIT) Chihuahua, en el cual se les explicó qué se hace en este lugar y cómo ayudan a las demás personas. Las primeras tres actividades se abordaron dentro de un tiempo de 30 minutos con recursos del Jardín de niños. Mientras que la plática fue de dos horas con 
ayuda del Instituto Chihuahuense de Lengua de señas mexicana, y la visita al CRIT todo el día. Estas últimas fueron de gran importancia, pues con ellas vivieron experiencias impartidas por profesionales, complementando aprendizajes para la vida, tanto futura como presente.

\section{Resultados}

Una de las cosas más importantes para el proyecto, es saber reflexionar sobre los resultados; los cuales, según Antonio Latorre, 2004 "son conjuntos de tareas con el fin de extraer significados relevantes, o pruebas en la relación con los efectos o consecuencias del plan de acción". Para valorar el resultado del proyecto se reflexionó de acuerdo con los aprendizajes de los niños y de las niñas indagando sus conocimientos respecto a los temas vistos al finalizar su aplicación.

Para conocer los resultados y el trabajo que se llevó a cabo durante el proyecto se tomó en cuenta el portafolio de evidencias. Gabriela Murillo cita a (Hernández, 2006) quién nos menciona que consiste en el "aporte de producciones de diferente índole por parte de quien es docente o de quien es estudiante". Es a través de estas evidencias que se pudo valorar el desempeño en el marco de una disciplina o curso específico, en este caso del proyecto

A su vez se utilizó la fotografía que, según M. de Miguel, J quien cita a Augusto Comte, (1839)“es una selección de datos, estudio de las fotografías, tiempo de integración y después del estudio de las fotos". Por lo que fue de gran utilidad al momento de justificar cada una de las actividades realizadas, así como poder identificar los avances que se lograron durante la implementación de las actividades. Además de tener cada una de las evidencias necesarias para obtener una postura y capturar lo que fue significativo para los alumnos durante el proyecto.

\section{Desglose de resultados}

Primer Día: Visual

Al comentarles que existían personas invidentes, los alumnos se sorprendieron y algunos expresaron empatía, queriendo dar apoyo a personas que padecen esta discapacidad.

En esta actividad los alumnos mostraron nervios, miedo y frustración al momento de caminar con los ojos vendados y realizar los dibujos.

Segundo día: Autismo 
Con uso del proyector, se les mostraron tres cuentos sobre el autismo y las diferentes perspectivas de verlo (en la casa, en la escuela y con los amigos). Al aplicarles la actividad de elaborar un dibujo o contar un cuento con ruidos y música fuerte, los alumnos se frustraron y su reacción inmediata fue taparse los oídos. Al explicarles que había personas que vivían con ese ruido todo el tiempo, se sorprendieron y el tiempo ahí se volvió emotivo, todos estaban en silencio, mostrando respeto y compasión hacía las personas que padecen autismo.

\section{Tercer día: Discapacidad física}

Los alumnos por parte de los tres grupos mostraron frustración y desesperación al no poder usar sus dos manos para trabajar.

Algunos comentaron que ya estaban cansados, dejando de lado la actividad.

En uno de los grupos, a la hora de ir por sus colores sin levantarse de su silla, uno de los alumnos no podía trasladarse a su lugar y una compañera al ver su frustración, se levantó a ayudarlo, mostrando empatía y servicio hacia los demás.

\section{Cuarto día: Discapacidad Auditiva}

Se dio la plática sobre la discapacidad auditiva donde se vio interés por parte de los alumnos y docentes, se perdió un poco la atención de los niños debido a la cantidad de alumnos que estaban presentes, el espacio en el que se encontraban y el clima no favoreció la explicación. Aun así, los alumnos aprendieron a comunicar algunas palabras en lenguaje signado.

\section{Quinto día: Vista al CRIT}

Los alumnos se sorprendieron al ver las instalaciones y conocer niños con diferentes discapacidades, algunos les sonreían y otros solo sentían tristeza.

\section{Conclusiones}

En la semana que se aplicó el proyecto, se presentaron una variedad de fortalezas, retos y barreras con los cuales se cumplieron la mayoría de los objetivos esperados.

Algunos de los alumnos mostraron mediante las actividades sobre las diferentes discapacidades, la empatía y sensibilidad que era esperada. Otros presentaban una actitud de juego y risa, sin comprender la importancia de los temas. Esto se vio reflejado en los diferentes ámbitos y no se vieron los resultados hasta el fin con la visita al CRIT en donde los mismos alumnos observaron como es que se daban las terapias y que ellos también podían ayudar a los demás formando de esta manera un ambiente de inclusión. 
RECIE. Revista Electrónica Científica de Investigación Educativa Vol. 4, núm. 2, enero-diciembre 2019, pp. 1029-1037.

Los retos se presentaron como momentos de ausencia con las maestras titulares, actitud negativa por parte de una de las docentes de la institución, donde se tuvo que hablar para aclarar la situación, a su vez, se dieron actitudes apáticas por parte de los padres de familia, por lo que se vio a fondo la falta de inclusión y aceptación hacia personas con capacidades diferentes, además, por parte del equipo se vio la falta de experiencias en proyectos inclusivos, como falta de organización en pequeños detalles, por ejemplo, quien iba a hablar frente al personal, explicación de los hechos, entre otros.

A pesar de esto, el objetivo principal se vio reflejado en ciertos alumnos, por lo que se animó a las maestras titulares a darle seguimiento al proyecto para fomentar un cambio en la comunidad comenzado por los niños, extendiéndose así, hacia los padres de familia. Pues hay que recordar que:

Al hablar de inclusión se habla tolerancia, respeto y solidaridad, pero sobre todo, de aceptación de las personas, independientemente de sus condiciones. Sin hacer diferencias, sin sobreproteger ni rechazar al otro por sus características, necesidades, intereses y potencialidades, y mucho menos, por sus limitaciones $\{. .$.$\} (Ronald, S.$ 2003).

Por lo cual se debe empezar con la sensibilización para dar paso a la cultura de la inclusión, en la que influya toda la sociedad, y en que mejor lugar empezar que con los alumnos y docentes en las aulas, para que de esta forma tenga un mayor alcance y se logre un mayor objetivo.

\section{Referencias}

Latorre, A. (2005), La investigación-acción conocer y cambiar la práctica educativa, Edit. Graó, Barcelona.

M. de Miguel, J. "Para una sociología de la Fotografía", Recuperado de: http://reis.cis.es/REIS/PDF/REIS_084_08.pdf.

Muñoz Corvalán, J.: "La cultura en la sociedad actual", en Contribuciones a las Ciencias Sociales, Noviembre 2012, www.eumed.net/rev/cccss/22/

Murillo Sancho, Gabriela, EL PORTAFOLIO COMO INSTRUMENTO CLAVE PARA LA EVALUACIÓN EN EDUCACIÓN SUPERIOR. Revista Electrónica "Actualidades Investigativas en Educación" [en línea] 2012, 12 (Sin mes) : [Fecha de consulta: 2 de junio de 2018] 
Disponible

en:<http://www.redalyc.org/articulo.oa?id=44723363015> ISSN

SEP, (2011) Programa de Educación Preescolar 2011. México: SEP

Soto Calderón, Ronald, La inclusión educativa: Una tarea que le compete a toda una sociedad . Revista Electrónica "Actualidades Investigativas en Educación" [en linea] 2003, 3 (enero-junio) : [Fecha de consulta: 13 de septiembre de 2018] Disponible en:<http://www.redalyc.org/articulo.oa?id=44730104> ISSN

Trilla J. (1998), Animación Sociocultural, Teorías, programas y ámbitos, $2^{\underline{a}}$ edición. Edit. Ariel, México.

Verdugo Alonso, M. (2017) Derechos y calidad de vida en personas con discapacidad intelectual y mayores necesidades de apoyo. Edit. Universidad de Salamanca, Salamanca Recuperado de: https://www.researchgate.net/profile/Patricia_Navas/publication/3 24889833_Derechos_y_calidad_de_vida_en_personas_con_discapacid ad_intelectual_y_mayores_necesidades_de 
RECIE. Revista Electrónica Científica de Investigación Educativa Vol. 4, núm. 2, enero-diciembre 2019, pp. 1029-1037. 